\title{
Plasmapheresis for the management of acute cyanide poisoning : A case report and review of literature
}

Navid Namakizadeh Esfahani ${ }^{1}$, Shafeajafar Zoofaghari ${ }^{1}$, Amirhossein Akhavan Sigari ${ }^{1}$, and Gholamali Dorooshi ${ }^{1}$

${ }^{1}$ Isfahan University of Medical Sciences

January 11, 2021

Plasmapheresis for the management of acute cyanide poisoning : A case report and review of literature

Navid Namakizadeh Esfahani

Isfahan University of Medical Sciences, Isfahan, Iran

navidnamakizadeh@gmail.com

Shafeajafar Zoofaghari

Department of Clinical Toxicology, Isfahan Clinical Toxicology Research Center, Khorshid hospital, Isfahan, Iran

shafeajafar@med.mui.ac.ir

Amirhossein Akhavan Sigari (corresponding author)

Isfahan University of Medical Sciences, Isfahan, Iran

amirsigari@aol.com

Tel: +989132151729

PO: 8174673461

Gholamali Dorooshi

Department of Clinical Toxicology, Isfahan Clinical Toxicology Research Center, Khorshid hospital, Isfahan, Iran

gdorvashy@med.mui.ac.ir

\section{Keywords}

Plasmapheresis

Cyanide toxicity

Toxicology

Emergency Medicine

Key clinical message 
In cases of mild to moderate cyanide poisoning, plasmapheresis can be utilized as an alternative option alongside supportive measures and when standard antidote kits are not readily available.

\section{Introduction}

Cyanide poisoning is a well known intoxication and a historically recognized cause of suicide.(1) Cyanide salts are widely used in the jewelry industry and are not readily available to the public. Ten percent of the causes of intoxication and suicide involve cyanide salts. (2,3) By disrupting the enzymatic pathways involved in cellular respiration, cyanide hinders utilization of oxygen by cells leading to their rapid dysfunction. Cyanide-induced hypoxia ultimately leads to neurologic and cardiovascular compromise. (4)

Rapid diagnosis of cyanide poisoning may not be possible due to the nonspecific presentations and the limited availability of blood cyanide level kits and their time consuming nature. Headache, confusion, vertigo, palpitations, respiratory distress and hyperventilation are among the manifestations of acute cyanide intoxication. Rapid development of hypotension, shock, bradycardia, and ultimately death may ensue. (4) Cellular hypoxia leads to acid-base disorders, and lactic acidosis is a hallmark of cyanide intoxication. Several studies have pointed to lactate levels as a sensitive marker in cyanide poisoning. (4)

Management of cyanide poisoning mainly comprises of supportive measures for preserving organ perfusion and maintaining proper homeostasis by correction of acid-base disorders and use of vasopressors and hydration in cases of severe hypotension. Specific antidote kits are used in emergency settings however, these kits may not readily be available at all centers. Other measures such as hemodialysis and plasmapheresis may be used on a case by case basis to eliminate toxic substances from the circulation. $(2,5)$

In this report we present a case of an intentional cyanide ingestion who was brought to our emergency department. Aside from supportive measures taken, due to the unavailability of cyanide antidote kits at our center, plasmapheresis was initiated.

\section{Case presentation}

A 39 year old male patient was brought to the emergency department with severe nausea and vomiting proceeding the intentional ingestion of two spoons full of cyanide salt that was obtained from a friend working in the jewelry industry. The time of ingestion was seven hours before admittance. Immediately after the ingestion of the powder, gastric irrigation and activated charcoal administration was undertaken in a primary health care facility and was then referred to our emergency department due to the development of refractory nausea and severe vomiting.

Upon arrival the patient has an oral temperature of 37.2 degrees Celsius, pulse rate of 110 beats per minute, respiratory rate of 18 breaths per minute, blood pressure recording of 110/70 and a Glasgow coma scale (GCS) score of 15. Pulse oximetry showed 97\%. Peripheral perfusion assessment was Physical examination other than an evident tachycardia was otherwise normal. The patients reported tobacco use but no history of alcohol abuse or addiction to illicit drugs.

After admittance to the emergency department primary blood work revealed leukocytosis and metabolic acidosis (lab data are summarized in table 1). ECG of the patient showed normal sinus rhythm and tachycardia.

An intravenous line was obtained and hydration and other supportive measures were started. After hours blood pressure recordings dropped to $67 / 35$ and peripheral perfusion was decreased. Due to the history obtained from the patient and the metabolic acidosis and hypotension, cyanide poisoning was suspected. As cyanide antidote kits were not available at our center, management consisted of norepinephrine 10-20 $\mathrm{mcg} / \mathrm{min}$, bicarbonate, and 2 sessions of plasmapheresis. Each plasmapheresis session was 3 hours. In the first session 3 liters of plasma were replaced with $2300 \mathrm{cc}$ normal saline and $700 \mathrm{cc}$ fresh frozen plasma and in the second session 3 liters of plasma was replaced with $2200 \mathrm{cc}, 700 \mathrm{cc}$, and 2 vials of normal saline, FFP, and albumin respectively. 
After the completion of plasmapheresis sessions hypotension and acidosis were resolved. VBG measurements are summarized in table 2 . The patient recovered completely after two days and was discharged.

\section{Discussion}

Early recognition of cyanide toxicity in the emergency department is of utmost importance. Patients with cyanide toxicity can present with a variety of manifestations based on the amount consumed, the time from consumption, and the route of exposure. In this manuscript we have presented a case of a 39 year old man who presented with acute cyanide poisoning and was treated with plasmapheresis in adjunct to other supportive measures.(6)

Although cyanide exposure can occur unintentionally and in occupational settings such as mining, agriculture, smoke, and metal industries, intentional ingestion of cyanide salts have been historically used for suicide and murder. Although the clinical presentations and laboratory data may not be specific at the time of presentation, some patients such as our case may confess of having intentionally ingested toxic cyanide salts.(3)

Patients with exposure to cyanide can present with altered levels of consciousness, seizures, respiratory compromise, nausea, vomiting, cardiac arrhythmias and arrest, blood pressure changes, altered body temperature, etc. All these symptoms arise secondary to cellular anoxia caused by cyanide, therefore organs with a high rate of oxygen consumption such as the brain, the heart, and the kidneys are prominently susceptible to damage. Although cherry red skin and bitter almond odor may be more specific of cyanide toxicity, they are only present in a minority of patients $(\sim 15 \%) \cdot(6,7)$

Primary management of cyanide poisoning is similar to the management of other toxicities; supportive care. Oxygen supplementation, hydration, and removal of the toxic source are the mainstay of therapy. Patients' vital signs and cardiac function should be monitored continuously. Intubation and vasopressors are inevitable if respiratory compromise or refractory hypotension occur, respectively. When available a cyanide antidote kit which consists of sodium thiosulfate, sodium nitrite, and amyl nitrite. Amyl nitrite and sodium nitrite cause the dissociation of cyanide from the cellular cytochrome oxidase, leading to cellular relief and the reversion to aerobic metabolism. The methemoglobinemia secondary to the use of nitrites will then be resolved by sodium thiosulfate. The end result is the excretion of thiocyanate in the urine.(6)

In our case, aside from vigilant supportive management, due to the unavailability of the cyanide antidote kit, we utilized plasmapheresis for intoxication. Plasmapheresis is not a common measure in cases of cyanide poisoning, however there have been seldom reports of plasmapheresis in such cases.

Z. Liu et al.(2) has reported a case of a 24 year old male patient who was diagnosed with concurrent methanol and cyanide poisoning. The patient has presented with bradycardia, hypotension, and decreased consciousness. Aside from supportive management, the patient was started on plasmapheresis 3 hours postingestion. The procedure was 2 hour in duration during which $2400 \mathrm{cc}$ plasma was removed from the patient with $3000 \mathrm{cc}$ of replacement solution consisting of $2000 \mathrm{cc}$ of FFP and $1000 \mathrm{cc}$ of NS. The patient had undergone hemodialysis after plasmapheresis. In our case the patient did not have any concurrent poisoning besides cyanide ingestion and did not undergo hemodialysis.

In another case Shatila et al.(8) has introduced a case of a young individual who presented to the emergency department with low oxygen saturation and increased methemoglobinemia. Supportive measures were taken and the patient was started on plasmapheresis on the second day of admission, however, primarily due to suspected thrombotic thrombocytopenic purpura (TTP). Plasmapheresis continued for six days during which after methemoglobin levels normalized. Although cyanide toxicity does not directly cause methemoglobinemia, the latter can be induced by the use of nitrites in such cases. In cases when the cyanide antidote kits may not be complete, induction of methemoglobinemia and subsequent plasmapheresis may be an option.

\section{Conclusion}

Cyanide poisoning can present with nonspecific symptoms which can complicate the course of diagnosis and 
rapid therapy. The clinical course can vary from mild to severe. Early recognition of cyanide toxicity can help guide in treatment and improve outcomes. In our case, due to the unavailability of cyanide antidote kits, we chose plasmapheresis beside other supportive measures. Treatment guidance based on a case report is not possible, however, plasmapheresis can be done when standard treatment is not available or the patient is not responding to such therapy.

\section{Declaration of Interest}

The authors have no conflicts of interest to declare

\section{Acknowledgements}

We honor the patient's consent for the publication of this article.

\section{Author Contribution}

NNE, SZ, and GD presented the case and were involved in the management of the patients and gathered relevant information from the case. AAS, NNE, and SZ wrote the primary and final draft of the manuscript. The final article was read and approved by all authors for publication.

\section{Consent to Publish}

The patient's consent was taken for the publication of his data in a scientific journal.

\section{Consent to participate}

Informed consent was taken from the patient for the report of this case.

\section{Funding}

No funding was received for the production of this manuscript.

\section{References}

1. Chin RG, Calderon Y. Acute cyanide poisoning: A case report. J Emerg Med. 2000;18(4):441-5.

2. Liu Z, Sun M, Zhao H, Zhao M. Acute Self-Induced Poisoning with Sodium Ferrocyanide and Methanol Treated with Plasmapheresis and Continuous Renal Replacement Therapy Successfully. Med (United States). $2015 ; 94(21): 1-4$.

3. Coentrão L, Moura D. Acute cyanide poisoning among jewelry and textile industry workers. Am J Emerg Med [Internet]. 2011;29(1):78-81. Available from: http://dx.doi.org/10.1016/j.ajem.2009.09.014

4. Dorooshi G, Dorostkar A, Rahimi A, Zoofaghari S. An unusual acute cyanide intoxication. Adv Biomed Res. 2020;9.

5. Gracia R, Shepherd G. Cyanide poisoning and its treatment. Pharmacotherapy. 2004;24(10 II):1358-65.

6. Peddy SB, Rigby MR, Shaffner DH. Acute cyanide poisoning. Pediatr Crit Care Med. 2006;7(1):79-82.

7. Huzar TF, George T, Cross JM. Carbon monoxide and cyanide toxicity: Etiology, pathophysiology and treatment in inhalation injury. Expert Rev Respir Med. 2013;7(2):159-70.

8. Shatila W, Verma A, Adam S. Plasmapheresis in severe methemoglobinemia following occupational exposure. Transfus Apher Sci [Internet]. 2017;56(3):341-4. Available from: http://dx.doi.org/10.1016/j.transci.2017.02.003 